DOI: $10.5965 / 223811711522016144$

\title{
Levantamento de besouros copro-necrófagos (Coleoptera: Scarabaeidae: Scarabaeinae) do Bioma Pampa
}

\author{
Evaluation of dung beetles (Coleoptera: Scarabaeidae: Scarabaeinae) in the Pampa Biome

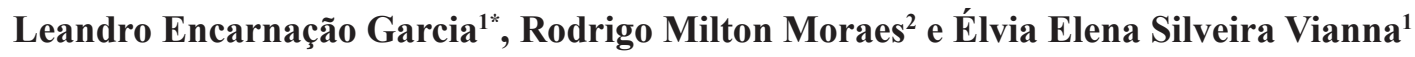 \\ Recebido em 04/02/2016 / Aceito em 26/04/2016
}

\section{RESUMO}

Scarabaeinae (Coleoptera: Scarabaeidae), compreende compreende os besouros popularmente chamados de "rola-bosta", que constituem importante grupo de animais detritívoros e bioindicadores de impactos ambientais nos ecossistemas. Áreas do Bioma Pampa são ecossistemas naturais com alta diversidade de espécies vegetais e animais, no entanto, pouco conhecidas em comparação com outros biomas do Brasil. Dados sobre a escarabeinofauna do Bioma Pampa é escassa. Portanto, os objetivos deste trabalho foram inventariar a fauna destes besouros em áreas localizadas no extremo-sul do Rio Grande do Sul, bem como, conhecer seus hábitos e comportamentos alimentares. As áreas de amostragem se localizaram nos municípios de Herval e Arroio Grande. Foram dispostas 216 armadilhas do tipo pitfall trap iscadas com esterco humano/suíno e carne bovina em decomposição, distribuídas em seis áreas. Registraram-se 2.243 indivíduos, organizados em 6 tribos, 13 gêneros e 35 espécies. As espécies foram classificadas em coprófagas, generalistas e necrófagas. Foi capturado um maior número de indivíduos em armadilhas iscadas com esterco suíno e humano do que em carne apodrecida. Pela primeira vez Trichillum morelli Verdú \& Galante, 1997 foi encontrada no Brasil e Canthidium taurinum Harold, 1867 é um novo registro para o estado.

PALAVRAS-CHAVE: detritívoros, rola-bosta, pitfall trap.

\footnotetext{
ABSTRACT

Scarabaeinae (Coleoptera: Scarabaeidae), comprises the beetles popularly called "dung beetle", which

${ }^{1}$ Universidade Federal de Pelotas, Pelotas, RS, Brasil.

${ }^{2}$ Universidade Federal do Rio Grande do Sul, Porto Alegre, RS, Brasil.

*Autor para correspondência <leandrogarcia20504@gmail.com>
}

are important group of animal detritivorous and biomarkers of environmental impacts on ecosystems. Pampa Biome areas are natural ecosystems with high diversity of plant and animal species, but are however, little known compared to other biomes of Brazil. Data on escarabeinofauna the Pampa Biome is scarce. Therefore, the aim of this study was to inventorize the fauna of these beetles in areas located in the far south of the Rio Grande do Sul, as well as learning their habits and eating behaviors. The sampling areas were located in the cities of Herval and Arroio Grande. 216 traps baited pitfall trap type with human dung and pig and beef decaying were placed distributed in six areas. They recorded 2,243 individuals, organized into six tribes, 13 genera and 35 species. The species were classified as coprophagous, general and scavenger. Greater number of subjects were caught in traps baited with human and pig manure than in rotting meat. For the first time Trichillum morelli Verdú \& Galante, 1997 was found in Brazil and Canthidium taurinum Harold, 1867 is a new record for the state.

KEYWORDS: detritivorous, dung beetles, pitfall trap.

\section{INTRODUÇÃO}

Os escarabeíneos (Coleoptera: Scarabaeidae: Scarabaeinae), compreendem os besouros popularmente conhecidos como "rola-bosta". Esses insetos são relacionados ao solo e utilizam-se dele para alocar recursos, abrigo e nidificação (HALFFTER \& MATTHEWS 1966). Os besouros rola-bostas realizam muitas funções ecológicas como ciclagem de nutrientes, aeração do solo, dispersão de sementes e controle biológico de espécies prejudiciais associadas ao gado (MITTAL 1993; NICHOLS et al. 2008). 
Essas são importantes atividades desempenhadas em larga escala no Bioma Pampa, área de estudo deste trabalho, contudo, dados sobre a fauna de besouros Scarabaeinae nestes ecossistemas são escassos (MORELLI \& GONZALES-VAINER 1997).

Estãodescritas aproximadamente 7.000 espécies de Scarabaeinae no mundo (SCHOOLMEESTERS et al. 2010). No Brasil, ocorre o registro de 700 espécies podendo aumentar a $1.200 \mathrm{com}$ a realização de novas coletas, revisões de gêneros e descoberta de novas espécies (VAZ-DE-MELLO 2000).

A maioria desses besouros é coprófaga, com alguma especificidade para estrume de grandes herbívoros, contudo há exceções, especialmente no sudeste Asiático e em toda a região Neotropical (HALFFTER 1991). Por este motivo, para a coleta destes besouros em estudos ecológicos, são indicadas armadilhas pitfall iscadas com fezes humanas, por serem consideradas mais atrativas e eficientes para escarabeíneos da região Neotropical (GARDNER et al. 2008). Este tipo de armadilha também é utilizado em estudos de diversidade, pois aumenta consideravelmente a eficiência amostral (HOWDEN \& NEALIS 1975; LOPES et al. 2006).

Conforme a utilização do esterco como recurso alimentar por esses besouros e seu tamanho corpóreo relativo, estes são organizados em três guildas comportamentais: endocoprídeos (residentes) ocupam a massa fecal e criam galerias nela, são as espécies menores; paracoprídeos (escavadores) alojam porções de fezes em túneis logo abaixo da placa fecal; e os telecoprídeos (roladores) rolam pelotas de esterco para longe da placa fecal, antes de enterrá-las, sendo compostos por indivíduos maiores (FLECHTMANN et al. 1995).

$\mathrm{O}$ estado do RS se encontra entre os mais carentes de levantamentos de scarabaeinofauna, com somente 79 espécies citadas, sendo cinco endêmicas. Este número está bem abaixo dos encontrados em estados próximos da região sul e sudeste do Brasil (VAZ-DE-MELLO 2000). No âmbito geral, os estudos de invertebrados também são precários no Rio Grande do Sul, sendo os lepidópteros e himenópteros os grupos mais estudados (SILVA et al. 2008). Dessa forma, esse estudo visa contribuir com informações sobre a fauna de besouros Scarabaeinae. Os objetivos deste trabalho foram inventariar a fauna de besouros Scarabaeinae em áreas de campos naturais do sudeste do Rio Grande do Sul; verificar abundância e riqueza das espécies amostradas e conhecer os hábitos e comportamentos alimentares das espécies registradas nestes habitats.

\section{MATERIAL E MÉTODOS}

\section{Área de Estudo}

As áreas de amostragem (Tabela 1) abrangem a porção sul do Bioma Pampa no Rio Grande do Sul, pertencentes aos municípios de Herval e Arroio Grande (Figura 1). Foram selecionadas seis áreas de campos naturais (vegetação nativa) sem perturbação, com pecuária extensiva (lotação de bovinos) onde havia também presença de equinos e ovinos. As áreas tinham extensão mínima de 50 hectares.

O extremo-sul do estado caracteriza-se por apresentar áreas de formações campestres naturais com altitude variando de 60 a $350 \mathrm{~m}$. Na classificação de Köppen, estas regiões apresentam clima subtropical com temperatura média dos meses mais quentes superiores a $22{ }^{\circ} \mathrm{C}(\mathrm{Cfa})$ com chuvas todos os meses (MORENO 1961). As amostras foram obtidas de seis áreas com distância mínima de $2 \mathrm{~km}$. As áreas pertencentes ao município de Arroio Grande (A1, A2 e A3) distanciaram-se $50 \mathrm{~km}$

Tabela 1 - Coordenadas geográficas das áreas de coletas selecionadas para o inventariamento das espécies de besouros Scarabaeinae em campos do sudeste do Rio Grande do Sul, Brasil.

Table 1 - Geographical coordinates of the areas selected for the collection of inventory the species of beetles Scarabaeinae in southeastern fields of Rio Grande do Sul, Brazil.

\begin{tabular}{|c|c|c|}
\hline Município Próximo & Área & Coordenadas \\
\hline \multirow{3}{*}{ Arroio Grande } & A1 & $32^{0} 13^{\prime} 532^{\prime \prime} \mathrm{S} ; 53^{0} 12^{\prime} 440^{\prime \prime} \mathrm{W}$ \\
\hline & $\mathrm{A} 2$ & $32^{0} 13^{\prime} 836^{\prime \prime} \mathrm{S} ; 53^{0} 14^{\prime} 145^{\prime \prime} \mathrm{W}$ \\
\hline & A3 & $32^{0} 14^{\prime} 053^{\prime \prime} \mathrm{S} ; 53^{0} 10^{\prime} 352^{\prime \prime} \mathrm{W}$ \\
\hline \multirow{3}{*}{ Herval } & A4 & $32^{0} 05^{\prime} 855^{\prime \prime} \mathrm{S} ; 53^{0} 36^{\prime} 837^{\prime \prime} \mathrm{W}$ \\
\hline & A5 & $32^{0} 05^{\prime} 373^{\prime \prime} \mathrm{s} ; 53^{0} 37^{\prime} 390^{\prime \prime} \mathrm{W}$ \\
\hline & A6 & $32^{0} 05^{\prime} 933^{\prime \prime} \mathrm{S} ; 53^{0} 36^{\prime} 959^{\prime \prime} \mathrm{W}$ \\
\hline
\end{tabular}




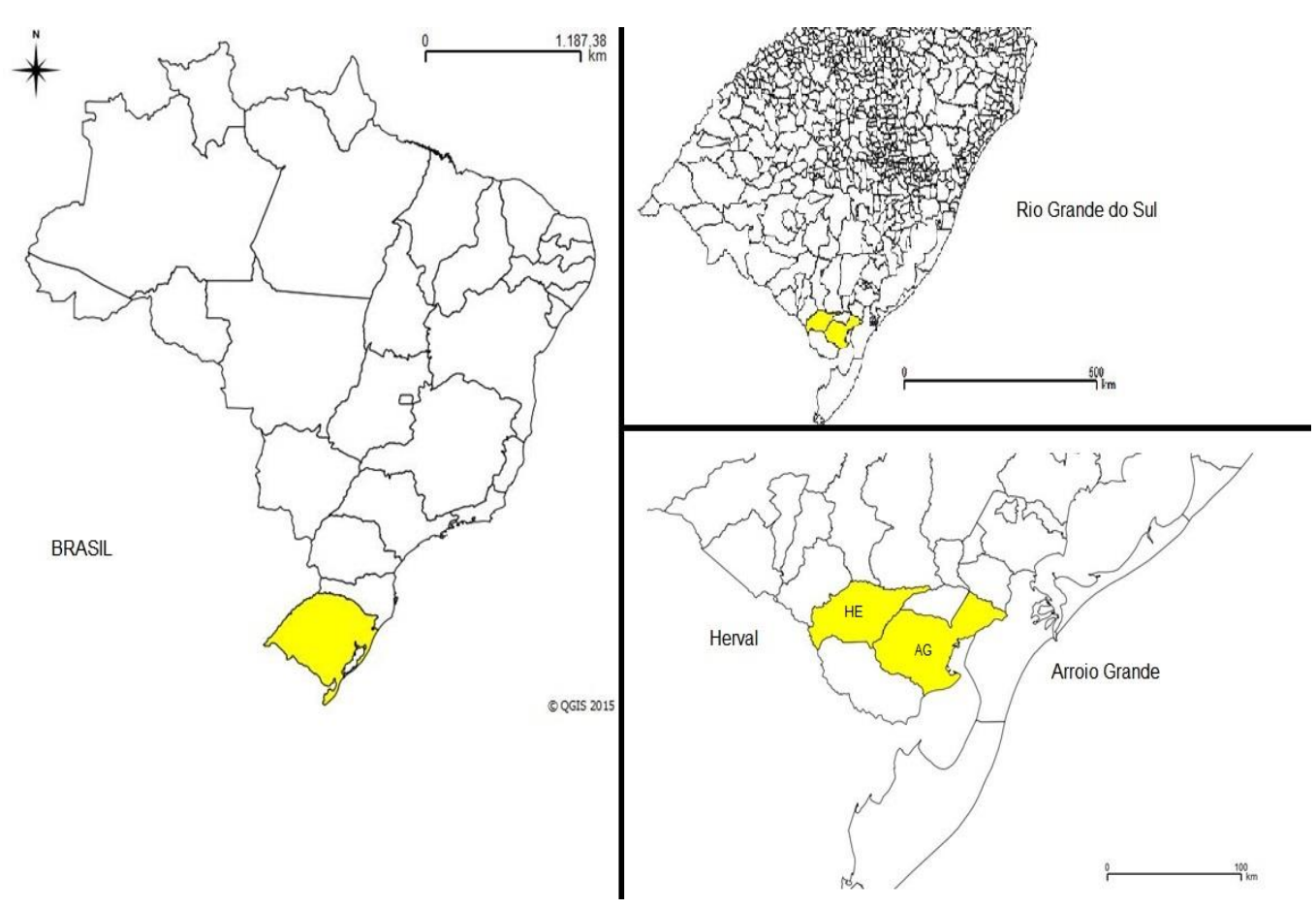

Figura 1 - Localização dos municípios de Herval e Arroio Grande no mapa do estado do Rio Grande do Sul.

Figure 1 - City locations of Herval and Arroio Grande on the map of Rio Grande do Sul.

das pertencentes ao município de Herval (A4, A5 e A6) (Figura 2). Cada uma das seis áreas de campos naturais com pecuária foi dividida em três lotações (réplicas espaciais), totalizando 18 lotações. As doze armadilhas de cada lotação foram organizadas em três quadrantes hipotéticos, onde nas arestas do quadrado foram montadas armadilhas de solo do tipo pitfall, distanciando-se entre si 40 metros. Em cada quadrante, metades das armadilhas de solo foram iscadas com carne em decomposição (baços bovinos expostos durante dois dias ao sol) e outra metade com esterco suíno e humano (1:1). Ao total do estudo foi utilizado 216 armadilhas do tipo pitfall. As coletas foram realizadas na primavera, estação com maior riqueza e abundância de besouros (SILVA 2011b) no mês de dezembro de 2013 e dezembro de 2014. A utilização da isca escolhida (esterco suíno e humano) objetiva a atração e captura do máximo de espécies de besouros rola-bostas presentes no local, não se restringindo a grupos especialistas de outros tipos de iscas, por exemplo, esterco bovino (MILHOMEM et al. 2003; FILGUEIRAS et al. 2009; HERNÁNDEZ \& VAZ-DE-MELLO 2009; SILVA et al. 2012c).

\section{Obtenção e análise dos dados}

A armadilha pitfall (Figura 3) é composta de um copo plástico de $500 \mathrm{ml}$ enterrado ao nível do solo, contendo formalina $5 \%$, e $20 \mathrm{ml}$ de detergente para quebrar a tensão superficial da solução. A isca foi colocada acima do pote enterrado ao nível do solo, dentro de um pote plástico menor de $50 \mathrm{ml}$. Para proteção das armadilhas foram utilizados pratos de plástico sobre as mesmas, onde permaneceram em campo por cinco dias, após esse período, foram recolhidas para triagem e identificação dos espécimes em laboratório. A triagem e identificação preliminar dos besouros contaram com o auxílio de estereomicroscópio, utilização da chave dicotômica para gêneros de Scarabaeinae (VAZ-DE-MELLO et al. 2011) e comparação com espécimes identificados por especialistas. Os exemplares foram depositados na coleção do Laboratório de Entomologia da Universidade Federal de Pelotas, Pelotas, RS. Para a classificação dos hábitos alimentares das espécies de Scarabaeinae, foi estabelecida a incidência de queda mínima de $80 \%$ nas armadilhas iscadas com os diferentes recursos (ALMEIDA \& LOUZADA 2009), e comparadas com dados de literatura (MARTÍNEZ 1959; HALFFTER \& MATTHEWS 1966). Dessa forma foi possível determinar a guilda trófica das espécies classificando-as em coprófagas (maior ocorrência na armadilha iscada com fezes humanas e suínas) e necrófaga (maior ocorrência na armadilha iscada com baço de bovino em decomposição) 


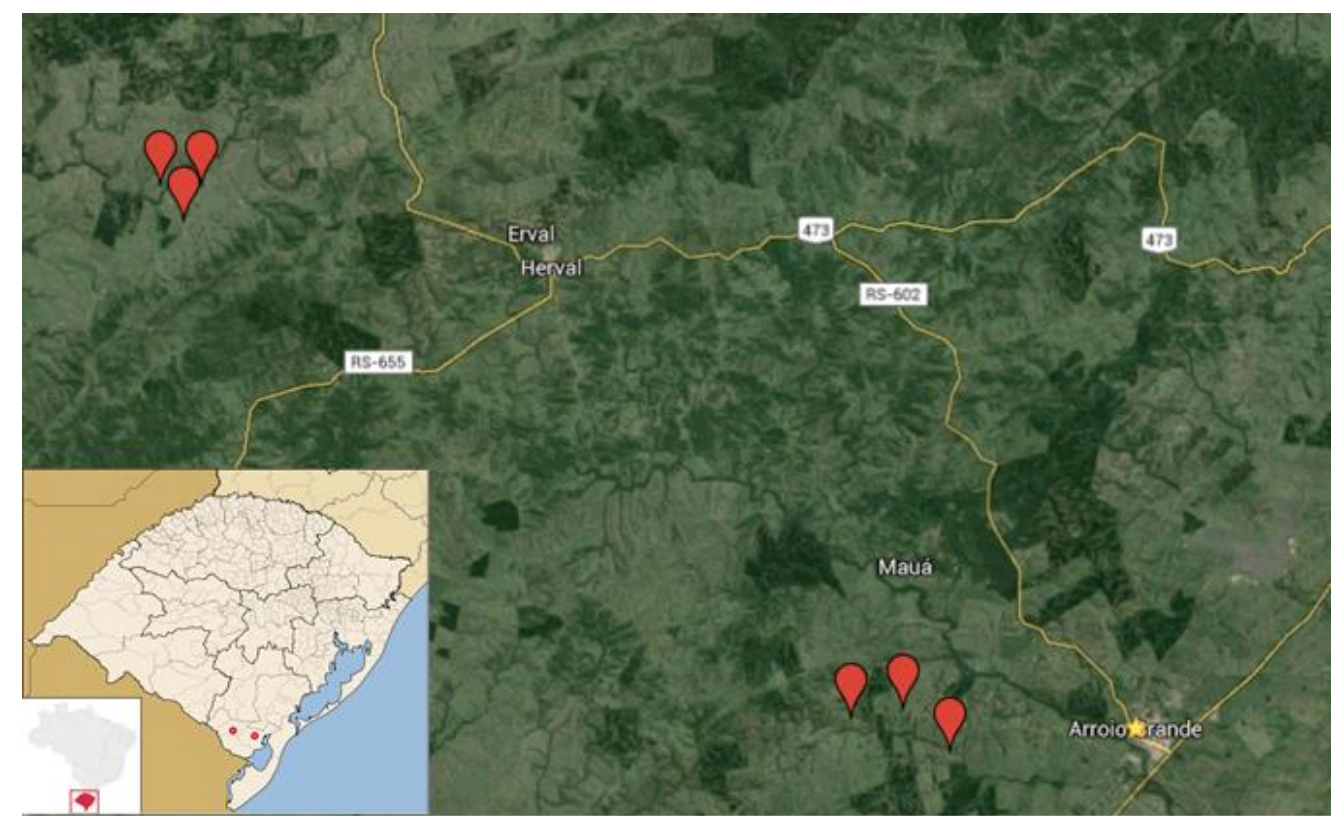

Figura 2 - Localização das áreas amostrais nos municípios de Herval e Arroio Grande em vermelho no mapa do estado do Rio Grande do Sul, Brasil.

Figure 2 - Location of sampling areas in the municipalities of Herval and Arroio Grande in red on the map of the state of Rio Grande do Sul, Brazil.

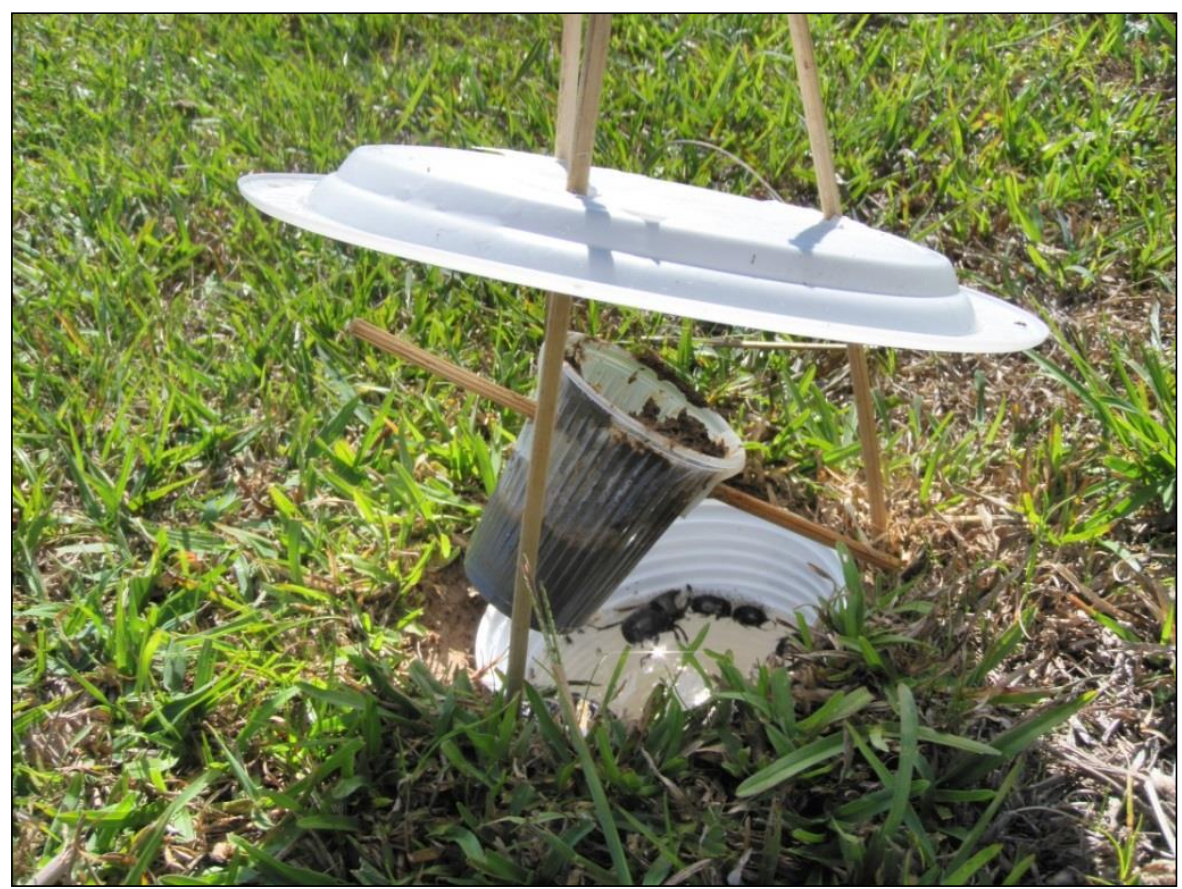

Figura 3 - Armadilha de solo (pitfall traps) para coleta de besouros rola-bostas em campos do sudeste, Bioma Pampa, Rio Grande do Sul, Brasil.

Figure 3 - Soil trap (pitfall traps) to collect dung-beetles in southeastern fields, Pampa Biome, Rio Grande do Sul, Brazil. 
(HALFFTER \& FAVILA 1993; HALFFTER \& ARELLANO 2002). Foram excluídas desta análise as amostras com um indivíduo e com dois indivíduos, pois não tiveram abundância suficiente de exemplares.

As espécies foram classificadas de acordo com o modo que utilizam o recurso alimentar para a nidificação (guilda funcional): Residentes (endocoprídeas alimentam-se e nidificam no interior do recurso); Escavadoras (paracoprídeas, escavam galerias no solo ao lado ou abaixo do recurso) e roladoras (telecoprídeas, retiram pequenas porções do recurso que são transformadas em esferas e roladas por diferentes distâncias até serem enterrados no solo) (HALFFTER \& MATTHEWS 1966; HANSKI \& CAMBEFORT 1991). A eficiência da amostragem foi avaliada através da curva de acumulação de espécies e seu intervalo de confiança, onde se relacionou as amostras com a riqueza observada. Foram utilizados os estimadores analíticos de riqueza Jackknife 1, Jackknife 2 e Bootstrap para verificar a representatividade das assembleias de besouros nas áreas estudadas. Estes estimadores são baseados na incidência de espécies por amostras, sendo os mais confiáveis para poucas amostras. A dominância foi estimada segundo o índice de Simpson (1-D) e a diversidade de acordo com o índice de ShannonWiener (H). A abundância e riqueza dos locais, dos hábitos e comportamentos alimentares foram comparadas através do teste de ANOVA um critério, onde foram utilizados os valores de abundância e riqueza total de cada lotação. Foi realizado o teste análise de similaridade (ANOSIM) para verificar diferença na composição de espécies entre os locais. Todas as análises foram realizadas através do programa Past versão 2.17 (HAMMER et al. 2001).

\section{RESULTADOS}

Foram coletados 2.243 indivíduos da subfamília Scarabaeinae, organizados em seis tribos, 13 gêneros e 35 espécies em campos do sudeste do Rio Grande do Sul, Brasil (Tabela 2). As espécies mais abundantes variam de acordo com as áreas: Deltochilum sculpituratum Felsche, 1907 (38,5\%) na área 1; nas áreas 2 e 3, a espécie Onthophagus hirculus Mannerheim, 1829(41,8\% e 63,3\%, respectivamente); na área 4, Canthidium breve Germar, 1824 (30,6\%) e nas áreas 5 e 6 Canthon rutilans Laporte, 1840 (57,6\% e $23,3 \%$, respectivamente).

As espécies mais frequentes em A1 foram $D$. sculpituratumn, O. hirculus e Canthon podagricus Harold, 1868, totalizando 70,5\%. Na área 2, O. hirculus, D. sculpituratum, Deltochilum elevatum Castelnau, 1840 e Canthon muticus Harold, 1868, foram as mais frequentes totalizando $81,9 \%$ das espécies. $\mathrm{Na}$ área 3, O. hirculus e Trichillum externepunctatum Borre, 1886 foram as mais frequentes com $79,9 \%$. Na área 4, C. breve, D. sculpituratum, Canthon lividus Blanchard, 1845, C. podagricus e O. hirculus foram as mais frequentes com $77,4 \%$. Na área 5 C. rutilans, Ontherus sulcator Fabricius, 1775, Uroxys sp. 1 e $D$. sculpituratum foram as mais frequentes com $77,9 \%$. Na área 6, C. rutilans, C. breve, D. sculpituratum e $C$. lividus foram mais frequentes com $65 \%$.

De acordo com os estimadores de riqueza (Tabela 3) Jackknife 1, Jackknife 2 e Bootstrap, em A1 $74-90 \%$ da assembleia foi amostrada, A2 77-88,9\%, A3 75-91\%, A4 75-92\%, A5 66,7-87\% e A6 77,8$91 \%$ a riqueza observada está próxima das estimadas. As curvas de rarefação de espécies baseadas nas amostras estão próximas de atingir a estabilidade (Figura 4). Entre os habitats amostrados a área dois (A2) mostrou o número mais elevado de espécies ( $\mathrm{S}=24, \mathrm{~N}=476)$ e $\mathrm{A} 4$ o menor número de espécies e indivíduos amostrados ( $\mathrm{S}=12$ e $\mathrm{N}=62$ ) (Tabela 2), no entanto, a área $\mathrm{A} 6$ teve o maior valor para o índice de Shannon $(2,344)$ similar ao valor obtido para a A2 $(2,12)$. O teste de ANOVA para comparação de riqueza e abundância na variável localidade não se mostrou significativo. Para a composição de espécies, o teste geral de ANOSIM foi significativo $(\mathrm{R}=0,3255 ; \mathrm{p}=0,0062)$, as áreas diferem com relação a sua composição de espécies. No mesmo teste de similaridade comparando as áreas de municípios próximos (Arroio Grande x Herval) o teste também se mostrou significativo $(R=0,53 ; p>0,0001)$.

Quatro áreas possuem espécies exclusivas, A2; Canthon sp. 2 e Dichotomius sp.1; A3, Dichotomius nisus Olivier, 1789 e Trichilum morelli Verdú \& Galante, 1997; A5, Canthon sp.5; A6, Canthon sp.6. Das espécies registradas somente $14 \%(\mathrm{~S}=5)$ foram compartilhadas em todas as áreas.

De acordo com a classificação dos hábitos alimentares (coprófagos, necrófagos e generalistas) 11 espécies foram classificadas como coprófagas (31,4\%), o mesmo número de espécies generalistas $(31,4 \%)$ e cinco espécies classificadas como necrófagas (14,3\%) (Tabela 4), as demais espécies $(22,9 \%)$ foram descartadas por terem sido coletadas uma ou duas vezes. 
Tabela 2 - Espécies de besouros Scarabaeinae amostrados em seis áreas de campos no Bioma Pampa com armadilha iscada com fezes suínas e humanas (1:1) e carne apodrecida, em dez/2013 e dez/2014, localizadas no sudeste do Rio Grande do Sul. S: número de espécie; N:número de indivíduos.

Table 2 - Scarabaeinae beetle species sampled in six areas of fields in the Pampas biome with trap baited with suine and human faeces (1:1) and rotting flesh, in Dec/2013 to Dec/2014, located in the southeast of Rio Grande do Sul. S.: number of species; N: number of individuals.

\begin{tabular}{|c|c|c|c|c|c|c|c|}
\hline \multirow{2}{*}{ Tribo/espécies } & \multicolumn{7}{|c|}{ Áreas } \\
\hline & 1 & 2 & 3 & 4 & 5 & 6 & Total \\
\hline ATEUCHINI $(\mathrm{S}=4 ; \mathrm{N}=199)$ & & & & & & & \\
\hline 1. Trichillum externepunctatum Borre, 1886 & 34 & 1 & 129 & & & & 164 \\
\hline 2. Trichillum morelli Verdú \& Galante, 1997 & & & 2 & & & & 2 \\
\hline 3. Uroxys dilaticolis Blanchard, 1845 & 1 & 3 & & & & & 4 \\
\hline 4. Uroxys sp. 1 & & 2 & & 1 & 16 & 9 & 28 \\
\hline COPRINI $(S=9 ; N=193)$ & & & & & & & \\
\hline 5. Canthidium breve (Germar, 1824) & 7 & 2 & & 19 & 2 & 61 & 91 \\
\hline 6. Canthidium chabanaudi Boucomont, 1928 & & 1 & 1 & & & & 2 \\
\hline 7. Canthidium moestum Harold, 1867 & 1 & 2 & & 3 & 5 & 5 & 16 \\
\hline 8. Canthidium taurinum Harold, 1867 & 1 & & & & 3 & 4 & 8 \\
\hline 9. Dichotomius sp. 1 & & 3 & & & & & 3 \\
\hline 10. Dichotomius sp. 2 & 4 & & 1 & & & 1 & 6 \\
\hline 11. Dichotomius sp. 3 & 2 & 6 & 3 & & & & 11 \\
\hline 12. Dichotomius nisus (Olivier, 1789) & & & 1 & & & & 1 \\
\hline 13. Ontherus sulcator Fabricius, 1775 & 4 & 5 & 21 & 3 & 16 & 6 & 55 \\
\hline DELTOCHILINI ( $\mathrm{S}=18 ; \mathrm{N}=1009)$ & & & & & & & \\
\hline 14. Canthon bispinus Germar, 1824 & 6 & & 1 & & & & 7 \\
\hline 15. Canthon lividus Blanchard, 1845 & 5 & 11 & 10 & 7 & 9 & 23 & 65 \\
\hline 16. Canthon mutabilis Lucas, 1857 & 1 & 8 & 2 & 2 & 1 & 2 & 8 \\
\hline 17. Canthon muticus Harold, 1868 & 10 & 35 & 15 & & 5 & 4 & $\begin{array}{l}32 \\
52\end{array}$ \\
\hline $\begin{array}{l}\text { 18. Canthon ornatus Burmeister, } 1873 \\
\text { 19. Canthon podagricus Harold, } 1868\end{array}$ & 62 & 9 & $\begin{array}{c}5 \\
21\end{array}$ & 7 & 4 & $\begin{array}{c}2 \\
13\end{array}$ & $\begin{array}{c}52 \\
116\end{array}$ \\
\hline $\begin{array}{l}\text { 19. Canthon podagricus Harold, } 1868 \\
\text { 20. Canthon rutilans Laporte, } 1840\end{array}$ & $\begin{array}{c}62 \\
1\end{array}$ & 12 & 21 & 2 & $\begin{array}{c}4 \\
128\end{array}$ & 62 & 205 \\
\hline 21. Canthon sp. 1 & & 8 & 4 & & & 1 & 13 \\
\hline 22. Canthon sp. 2 & & 2 & & & & & 2 \\
\hline 23. Canthon sp. 3 & & 2 & & & & 2 & 4 \\
\hline 24. Canthon sp.4 & & & & & & 2 & 2 \\
\hline 25. Canthon sp. 5 & & & & & 1 & & 1 \\
\hline 26. Canthon sp. 6 & & & & & 1 & & 1 \\
\hline 27. Canthon sp. 7 & & & 2 & & 1 & 2 & 5 \\
\hline 28. Deltochilum sculpituratum Felsche, 1907 & 170 & 114 & 33 & 9 & 13 & 26 & 365 \\
\hline 29. Deltochilum elevatum (Castelnau, 1840) & 18 & 42 & 11 & 0 & 1 & 17 & 89 \\
\hline 30. Malagoniella magnifica (Balthasar, 1939) & & 3 & 15 & 2 & 5 & 8 & 33 \\
\hline 31. Vulcanocanthon seminulus (Harold, 1867) & 2 & 4 & 2 & & 1 & & 9 \\
\hline ONITICELINI ( $\mathrm{S}=1 ; \mathrm{N}=4)$ & & & & & & & \\
\hline 32. Eurysternus aeneus Génier, 2009 & 1 & 1 & & & 2 & & 4 \\
\hline ONTHOPHAGINI $(\mathrm{S}=1 ; \mathrm{N}=826)$ & & & & & & & \\
\hline 33. Onthophagus hirculus Mannerheim, 1829 & 107 & 199 & 492 & 6 & 7 & 15 & 826 \\
\hline PHANAEINI(S=2; N=13) & & & & & & & \\
\hline 34. Coprophanaeus milon (Blanchard, 1845) & 4 & 1 & 6 & & & & 11 \\
\hline 35. Sulcophanaeus menelas (Castelnau, 1840) & & & & 1 & 1 & & 2 \\
\hline Total & 441 & 476 & 777 & 62 & 222 & 265 & \\
\hline
\end{tabular}


Tabela 3 - Riqueza, abundância, índices de diversidade, dominância, espécies exclusivas e estimadores de riqueza (Jackknife 1, Jackknife 2 e Bootstrap) para as espécies de besouros Scarabaeinae amostrados com armadilhas iscadas com fezes suínas e humanas (1:1) e carne em decomposição em dez/2013 e dez/2014 nos campos localizadas no sudeste do Rio Grande do Sul, Brasil.

Table 3 -Richness, abundance, diversity index, dominance, exclusive species and richness estimators (Jackknife 1, Jackknife 2 and Bootstrap) for the species of dung beetles sampled with traps baited with suine and human faeces (1: 1) and meat rotting in dec/2013 and dec/2014 in the fields located in the southeast of Rio Grande do Sul, Brazil.

\begin{tabular}{lccccccc}
\hline & A1 & A2 & A3 & A4 & A5 & A6 & Total \\
\hline Riqueza & 20 & 24 & 21 & 12 & 20 & 21 & 35 \\
Abundância & 441 & 476 & 777 & 62 & 222 & 265 & 2243 \\
Diversidade de Shannon-Wiener (H') & 1,836 & 1,911 & 1,4 & 2,12 & 1,744 & 2,344 & - \\
Dominância de Simpson (D) & 0,2362 & 0,248 & 0,4331 & 0,1582 & 0,3512 & 0,1383 & - \\
Exclusivas & 0 & 2 & 2 & 0 & 1 & 1 & - \\
Singletons & 6 & 4 & 4 & 2 & 7 & 4 & - \\
Doubletons & 2 & 5 & 4 & 3 & 2 & 4 & \\
Jackknife 1 & 25 & 31 & 26 & 15 & 27 & 26 & - \\
Jackknife 2 & 27 & 31 & 28 & 16, & 30 & 27 & - \\
Bootstrap & 22 & 27 & 23 & 13 & 23 & 23 & - \\
\hline
\end{tabular}
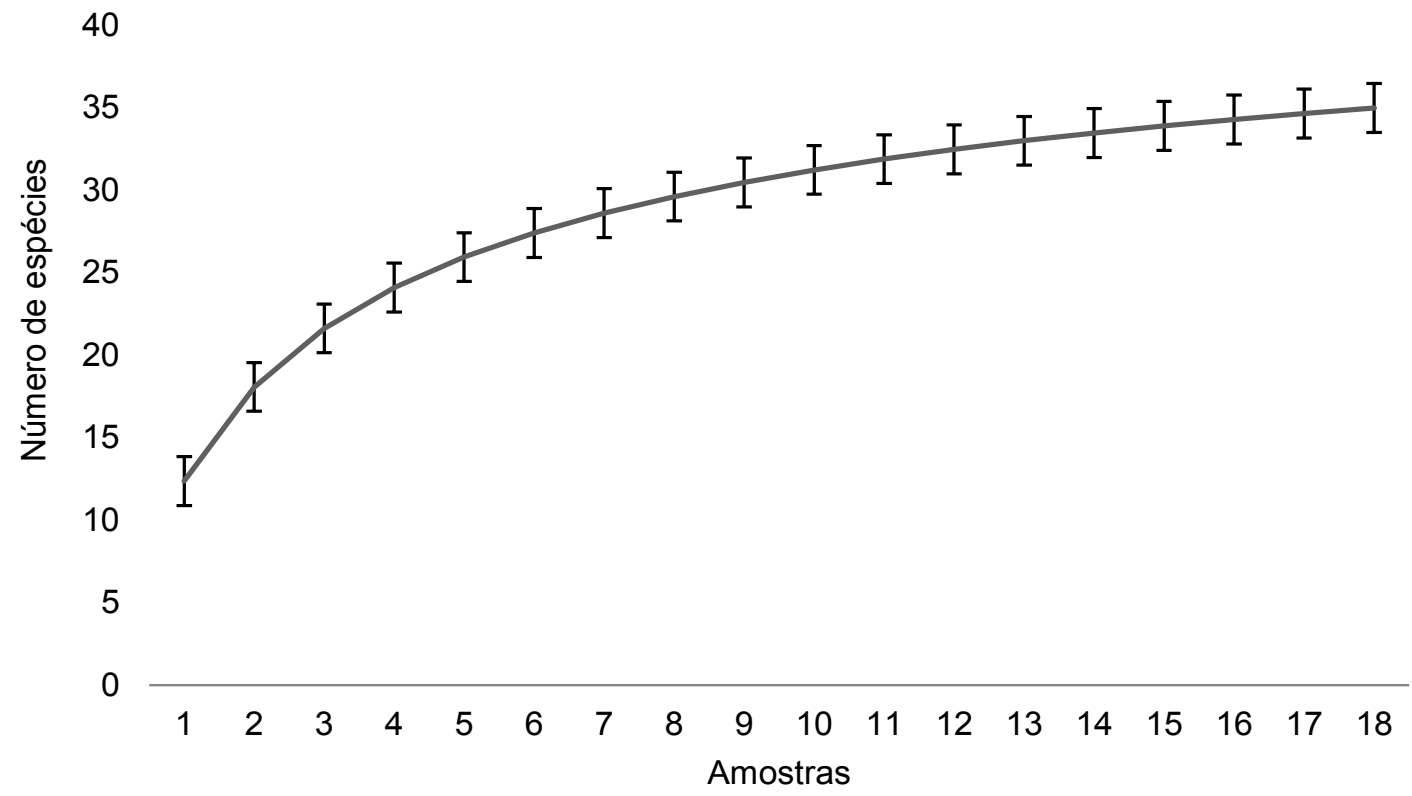

Figura 4 - Curva de rarefação baseado nas amostras para as espécies de besouros Scarabaeinae coletadas com armadilha pitfall iscadas com fezes suínas e humanas (1:1) e carne apodrecida em dez/2013 e dez/2014 em campos do sudeste do RS, Brasil.

Figure 4 - Rarefaction curve based on samples for species of beetles Scarabaeinae collected with pitfall trap baited with suine and human faeces (1:1) and rotting meat in dec/2013 and dec/2014 in in southeastern fields of RS, Brazil. 
Tabela 4 - Frequência absoluta das espécies de besouros amostrados em armadilhas iscadas com fezes suínas e humanas (ESH), carne em decomposição (CD) e classificada de acordo com a guilda trófica (GT) e guilda comportamental (GC) nos campos do sudeste do Rio Grande do Sul, Brasil de acordo com (MARTÍNEZ, 1959; HALFFTER \& MATTHEWS, 1966).

Table 4 - Absolute frequency of beetle species sampled in traps baited with suine and human faeces (ESH), rotting meat (CD) and classified according to the trophic guild (GT) and behavioral settings $(G C)$ in the southeastern fields of Rio Grande do Sul, Brazil according to (MARTÍNEZ 1959; HALFFTER \& MATTHEWS, 1966).

\begin{tabular}{|c|c|c|c|c|}
\hline Espécies & ESH & $\mathrm{CD}$ & GT & GC \\
\hline Canthidium breve & 87 & 4 & Coprófaga & Escavadora \\
\hline Canthidium chabanaudi & 0 & 2 & Doubleton & Escavadora \\
\hline Canthidium moestum & 5 & 11 & Generalista & Escavadora \\
\hline Canthidium taurinum & 7 & 1 & Coprófaga & Escavadora \\
\hline Canthon bispinus & 2 & 5 & Generalista & Roladora \\
\hline Canthon lividus & 7 & 58 & Necrófaga & Roladora \\
\hline Canthon mutabilis & 3 & 5 & Generalista & Roladora \\
\hline Canthon muticus & 18 & 14 & Generalista & Roladora \\
\hline Canthon podagricus & 33 & 83 & Generalista & Roladora \\
\hline Canthon rutilans & 100 & 105 & Generalista & Roladora \\
\hline Canthon ornatos & 47 & 5 & Coprófaga & Roladora \\
\hline Canthon sp. 1 & 10 & 3 & Generalista & Roladora \\
\hline Canthon sp. 2 & 2 & 0 & Doubleton & Roladora \\
\hline Canthon sp. 3 & 3 & 1 & Generalista & Roladora \\
\hline Canthon sp. 4 & 1 & 1 & Doubleton & Roladora \\
\hline Canthon sp. 5 & 0 & 1 & Singleton & Roladora \\
\hline Canthon sp. 6 & 0 & 1 & Singleton & Roladora \\
\hline Canthon sp. 7 & 3 & 2 & Generalista & Roladora \\
\hline Coprophanaeus milon & 1 & 10 & Necrófaga & Escavadora \\
\hline Dichotomius nisus & 1 & 0 & Singleton & Escavadora \\
\hline Dichotomius sp. 1 & 3 & 0 & Coprófaga & Escavadora \\
\hline Dichotomius sp.2 & 6 & 0 & Coprófaga & Escavadora \\
\hline Dichotomius sp. 3 & 11 & 0 & Coprófaga & Escavadora \\
\hline Deltochilum elevatum & 8 & 81 & Necrófaga & Roladora \\
\hline Deltochilum sculpituratum & 19 & 346 & Necrófaga & Roladora \\
\hline Eurysternus aeneus & 33 & 3 & Generalista & Residente \\
\hline Malagoniella magnifica & 29 & 4 & Coprófaga & Roladora \\
\hline Ontherus sulcator & 52 & 3 & Coprófaga & Escavadora \\
\hline Onthophagus hirculus & 701 & 123 & Coprófaga & Escavadora \\
\hline Sulcophanaeus menelas & 2 & 0 & Doubleton & Escavadora \\
\hline Trichillum externepunctatum & 142 & 24 & Coprófaga & Escavadora \\
\hline Trichillum morelli & 1 & 1 & Doubleton & Escavadora \\
\hline Uroxys aff. dilaticolis & 0 & 4 & Necrófaga & Escavadora \\
\hline Uroxys spp. & 13 & 15 & Generalista & Escavadora \\
\hline Vulcanocanthon seminulus & 9 & 0 & Coprófaga & Roladora \\
\hline
\end{tabular}


Foram capturados mais indivíduos em armadilhas iscadas com esterco suíno e humano, 1.329 indivíduos (59,2\%), do que em carne em decomposição com 914 indivíduos (40,8\%). O teste de ANOVA não foi significativo para a riqueza $(\mathrm{F}=0,8638 ; \mathrm{p}=0,5)$ e abundância das espécies $(\mathrm{F}=0,8279 ; \mathrm{p}=0,5) \mathrm{em}$ função dos hábitos alimentares. Foram capturadas 18 espécies roladoras (telecoprídeas), 16 espécies escavadoras (paracoprídeas) e uma espécies residente (endocoprídea). As espécies roladoras representam $45 \% \quad(\mathrm{~N}=1.009)$ dos indivíduos capturados, as escavadoras representam $54 \% \quad(\mathrm{~N}=1.230)$, sendo assim as mais abundantes nos ambientes amostrados. Teste de ANOVA não foi significativo para riqueza $(\mathrm{F}=0,1697 ; \mathrm{p}=0,96)$ e abundância $(\mathrm{F}=0,7104 ; \mathrm{p}=0,62)$ dos besouros classificados de acordo com o seu comportamento alimentar.

Todas as espécies roladoras pertencem à tribo Deltochilini, representadas por dois gêneros, Canthon Hoffmannsegg, 1817 e Deltochilum Eschscholtz, 1822. Canthon contendo 14 espécies e Deltochilum duas espécies, ambos amplamente distribuídos pela região Neotropical (VAZ-DE-MELLO 1999). Apenas uma espécie foi classificada como residente pertencendo à tribo Oniticelini, Eurysternus aeneus Génier, 2009, sendo as demais classificadas como paracoprídeas (escavadoras).

\section{DISCUSSÃO}

O número de espécies registradas nesse trabalho foi superior ao encontrado por SILVA et al. (2008) em áreas de potreiros no município de Bagé, RS, e AUDINO et al. (2011) em áreas com plantio de Eucalipto no município de Bagé, RS, onde registram, respectivamente, 18 e 28 espécies de escarabeíneos amostradas nestes ambientes. AUDINO et al. (2011) inferiram a hipótese de que o número de espécies em pastagens naturais poderia ser maior, o que é corroborado neste trabalho.

Levantamentos realizados por SILVA et al. (2007, 2008 e 2010) em Bagé, RS, registraram, respectivamente, 16,18 e 17 espécies para ecossistemas campestres. SILVA (2011a) identificou 13 espécies em duas diferentes áreas do sudeste do Rio Grande do Sul, e em 2012, amostraram O. hirculus como espécie mais abundante. Outro trabalho (SILVA \& DI MARE 2012b) reportou 14 gêneros distribuídos em 28 espécies em fragmentos florestais no centro do estado o Rio Grande do Sul, resultado este similar a esta pesquisa. Pode-se perceber que o número de espécies e a ampliação de registros de rola-bostas aumentam com o número de trabalhos, principalmente em áreas amostrais novas, pois muitos trabalhos com esses besouros se concentram na região central do estado.

A área A6 teve o maior valor para o índice de Shannon $(2,34)$ similar ao valor obtido para a A2 $(2,12)$. A menor dominância e maior equitabilidade das espécies nas áreas amostradas contribuem para o alto índice de diversidade Shannon nestes locais (MAGURRAN 1988; MORENO 2001). Além da combinação riqueza com uniformidade, o índice atribui maior peso para as espécies raras. Baixos valores de diversidade também podem estar associados aos locais com fatores limitantes de recurso e competição interespecífica. Essas situações costumam apresentar um maior número de espécies comuns e menor número de espécies raras, como podemos evidenciar em A3, onde $O$. hirculus foi a mais abundante de todas as áreas $(\mathrm{N}=492)$.

O número reduzido de espécies compartilhadas pelas áreas amostrais (Tabela 3) evidencia uma composição de espécies diferentes para cada uma dessas áreas, corroborado pelo teste de ANOSIM. Observando a curva de rarefação de espécies baseada nas amostras (Figura 4), o esforço amostral mostrase satisfatório, pois tende a estabilização, quase atingindo a assíntota.

Com relação às espécies observadas, os estimadores de riqueza sugerem que se estudos nestas áreas continuassem, provavelmente iriam ocorrer novos registros. Somando o total de espécies coprófagas e necrófagas $(\mathrm{S}=16)$, o número mostrouse maior que o de espécies generalistas $(S=11)$, resultado esse semelhante ao encontrado por SILVA et al. (2012a) e diferindo da hipótese que as assembleias neotropicais de besouros Scarabaeinae possuem proporção maior de besouros generalistas em relação aos especialistas (HALFFTER \& MATTHEWS 1966; HALFFTER 1991).

Um maior número de espécies roladoras é comum para ambientes de campo, assim como um maior número de espécies escavadoras é comum em ambientes típicos de floresta (HALFFTER G \& FAVILA 1993; LOUZADA \& LOPES 1997). Apesar de semelhantes, o número de espécies roladoras foi ligeiramente maior que as espécies escavadoras, talvez porque aquelas espécies tenham preferência por ambientes de áreas abertas e espécies escavadoras possam ser mais generalistas ao tipo de ambiente e 
espacialidade.

A maioria das espécies capturadas são comuns para a região central do Rio Grande do Sul (SILVA et al. 2012c). Segundo a primeira lista de espécies de Scarabaeinae para o estado (SILVA2011b), as espécies melhor distribuídas são: O. sulcator, Sulcophanaeus menelas Castelnau, 1840 e T. externepunctatum. Elas estão presentes em vários estados do Brasil e também registradas neste trabalho. A espécie Trichillum morelli é o primeiro registro para o Brasil, sendo de grande valia para conhecimento de diversidade da fauna Scarabaeinae, e Canthidium taurinum Harold, 1867 um novo registro para o estado.

Segundo os estimadores de riqueza, houve uma média de mais de $70 \%$ de indivíduos capturados por habitat amostrado. $\mathrm{O}$ número registrado de espécies $(\mathrm{S}=35)$ é maior inclusive em comparação com outros trabalhos realizados tanto em campos abertos como em fragmentos de matas do Bioma Pampa (AUDINO et al. 2011; SILVA 2011a; SILVA \& DI MARE 2012b; SILVA et al. 2013). A média de singleton e doubleton foi de 3,3 e 4,5 espécies por área, semelhante ao encontrado por SILVA et al. (2012a) também em campo nativo.

\section{CONCLUSÃO}

Foi obtido um número de 35 espécies de Scarabaeinae em áreas de campos do Bioma Pampa, no estado do Rio Grande do Sul, sendo O. hirculus a mais abundante para a região. A composição da fauna difere entre as seis áreas amostrais apesar da riqueza e abundância de espécies de Scarabaeinae não indicarem significância estatística. Não houve predominância entre os hábitos alimentares coprófagos e necrófagos das espécies de Scarabaeinae amostradas.

Este trabalho acrescenta dados importantes para fauna de Scarabaeinae em campos do sudeste no Bioma Pampa do Rio Grande do Sul, como o registro inédito da espécie T. morelli para o Brasil e o apontamento de um novo registro para o estado, $C$. taurinum. Obteve-se uma ampliação do conhecimento acerca da distribuição geográfica das espécies de Scarabaeinae no Bioma Pampa.

\section{AGRADECIMENTOS}

À Coordenação de Aperfeiçoamento de Pessoal de Nível Superior (CAPES), pela concessão de bolsa de nível de mestrado. Agradeço também aos revisores anônimos pelas valiosas sugestões.

\section{REFERÊNCIAS}

ALMEIDA SSP \& LOUZADA JNC. 2009. Estrutura da comunidade de Scarabaeinae (Scarabaeidae: Coleoptera) em fitofisionomias do cerrado e sua importância para a conservação. Neotropical Entomology 38: 32-43.

AUDINO LD et al. 2011. Scarabaeinae (Coleoptera, Scarabaeidae) de um bosque de eucalipto introduzido em uma região originalmente campestre. Iheringia. Série Zoologia 101: 121-126.

FILGUEIRAS BKC et al. 2009. Attractivity of omnivore, carnivore and herbivore mammalian dung to Scarabaeinae (Coleoptera, Scarabaeidae) in a tropical Atlantic rainforest remnant. Revista Brasileira de Entomologia 53: 422-427.

FLECHTMANN CAH et al. 1995. Controle Biológico da mosca-dos-chifres (Haematobia irritans irritans) em Selvíria, Mato Grosso do Sul. 3. Levantamento de espécies fimícolas associadas à mosca. Revista Brasileira de Entomologia 39: 249-258.

GARDNER TA et al. 2008. Understanding the biodiversity consequences of habitat change: the value of secondary and plantation forests for neotropical dung beetles. Journal of Applied Ecology 45: 883-893.

HAMMER O et al. 2001. Past: Paleontological statistics software package for education and data analysis. Palaeontologia Electronica 4: 1-9.

HALFFTER G \& MATTHEWS EG. 1966. The natural history of dung beetles of the subfamily Scarabaeinae. Folia Entomologica Mexicana. 312p.

HALFFTER G. 1991. Historical and ecological factors determining the geographical distribution of beetles (Coleoptera: Scarabaeidae: Scarabaeinae). Folia Entomologica Mexicana 82: 195-238.

HALFFTER G \& FAVILA ME. 1993. The Scarabaeidae (Insecta: Coleptera) an animal group for analyzing, inventorying and monitoring biodiversity in tropical rain forest and modified landscapes. Biology International 27: 15-21.

HALFFTER G \& ARELLANO L. 2002. Response of dung beetle diversity to human-induced changes in a tropical landscape. Biotropica 34: 144-154.

HANSKI I \& CAMBEFORT Y. 1991. Competition in dung beetles. HANSKI I \& CAMBEFORT Y. eds. Dung beetle ecology. Princeton: Princeton University Press. p. 305-329. HERNÁNDEZ MIM \& VAZ-DE-MELLO FZ. 2009. Seasonal and spatial species richness variation of dung beetle (Coleoptera, Scarabaeidae s. str.) in the Atlantic Forest of southeastern Brazil. Revista Brasileira de Entomologia 53: 607-613.

HOWDEN HF \& NEALIS VG. 1975. Effects of clearing in a tropical rain forest on the composition of the coprophagous scarab beetle fauna (Coleoptera). Biotropica 7: 77-83.

LOPES PP et al. 2006. Organization of dung beetle communities (Coleoptera: Scarabaeidae) in areas of vegetation re-establishment in Feira de Santana, Bahia, 
Brazil. Sitientibus Série Ciências Biológicas 6: 261-266. LOUZADA JNC \& LOPES FS. 1997. A comunidade de Scarabaeidae copro-necrófagos (Coleoptera) de um fragmento de Mata Atlântica. Revista Brasileira de Entomologia 41: 117-121.

MAGURRAN AE. 1988. Ecological diversity and its measurement. Princeton: Princeton University Press, 179p. MARTÍNEZ A. 1959. Catalogo de los Scarabaeidae Argentinos (Coleoptera). Revista del Museo Argentino de Ciencias Naturales 5: 1-126.

MILHOMEM MS et al. 2003. Técnicas de coleta de besouros copronecrófagos no Cerrado. Pesquisa Agropecuária Brasileira 38: 1249-1256.

MITTAL IC. 1993. Natural manuring and soil conditioning by dung beetles. Tropical Ecology 34: 150-159.

MORELLI E \& GONZALEZ-VAINER P. 1997. Dung beetles (Coleoptera; Scarabaeidae) inhabiting bovine and ovine droppings in Uruguayan prairies. The Coleopterists Bulletin 51: 197-203.

MORENO JA. 1961. Clima do Rio Grande do Sul. Porto Alegre: Secretaria da Agricultura, 42p.

MORENO CE. 2011. Método para medir la biodiversidade. M\&T-Manuales y Tesis SEA. Zaragoza: Gorfi, 84p.

NICHOLS E et al. 2008. Ecological functions and ecosystem services provided by Scarabaeinae dung beetles. Biological Conservation 141: 1461-1474.

SCHOOLMEESTERS P et al. 2010. ScarabNet Global Taxon Database (version 1.5). Disponível em: <www. researchgate.net/publication/258344500_ScarabNet_ Global_Taxon_Database_version_15>Acesso em: 02 jun. 2016.

SILVA PG et al. 2007. Besouros rola-bosta: insetos benéficos das pastagens. Revista Brasileira de Agroecologia 2: $1428-1432$.

SILVA PG et al. 2008. Espécies de besouros copronecrófagos (Coleoptera: Scarabaeidae) da Região da Campanha do Rio Grande do Sul. Revista de Ciências Agroveterinárias 7: 143-149.

SILVA PG et al. 2010. Besouros copro-necrófagos (Coleoptera: Scarabaeidae sensu stricto) do município de Bagé, RS (Bioma Campos Sulinos). Biociências 17: 33-43. SILVA PG. 2011a. Dung beetles (Coleoptera: Scarabaeidae: Scarabaeinae) of two non-native habitats in Bagé, Rio Grande do Sul, Brazil. Zoological Studies 50: 546-559.

SILVA PG. 2011b. Espécies de Scarabaeinae de Fragmentos Florestais com diferentes níveis de alteração em Santa Maria, Rio Grande do Sul. Dissertação (Mestrado em Ciências Biológicas). Santa Maria: UFSM. 168p.

SILVA PG. et al. 2012a. Escarabeíneos (Coleoptera: Scarabaeidae: Scarabaeinae) de uma área de campo nativo no bioma Pampa, Rio Grande do Sul, Brasil. Biota Neotropica 12: 246-253.

SILVA PG \& DI MARE RA. 2012b. Escarabeíneos copronecrófagos (Coleoptera, Scarabaeidae, Scarabaeinae) de fragmentos de Mata Atlântica em Silveira Martins, Rio
Grande do Sul, Brasil. Iheringia. Série Zoológica 102: 197205.

SILVA PG et al. 2012c. Attractiveness of different bait to the Scarabaeinae (Coleoptera: Scarabaeidae) in forest fragments in extreme southern Brazil. Zoological Studies 51: 429-441.

SILVA PG et al. 2013. Diversity and seasonality of Scarabaeinae (Coleoptera: Scarabaeidae) in forest fragments in Santa Maria, Rio Grande do Sul, Brazil. Anais da Academia Brasileira de Ciências 85: 679-697.

VAZ-DE-MELLO FZ. 1999. Scarabaeidae s. str. (Coleoptera: Scarabaeoidea) de um fragmento de Floresta Amazônica no Estado do Acre, Brasil. Anais da Sociedade Entomológica do Brasil 28: 447-453.

VAZ-DE-MELLO FZ. 2000. Estado atual do conhecimento de Scarabaeidae s. str. (Coleoptera: Scarabaeiodea) do Brasil. PriBES, Proycto ibero-americano de biogeografia y entomología sistemática 1: 183-195.

VAZ-DE-MELLO FZ et al. 2011. A multilingual key to the genera and subgenera of the subfamily Scarabaeinae of the New World (Coleoptera: Scarabaeidae). Zootaxa 2854: 1-73. 